\title{
Novel bipolar charge collecting structure enabling overall water splitting on ferroelectric photocatalysts
}

Yong Liu

Dalian Institute of Chemical Physics

\section{Zhuan Wang}

Institute of Physics, Chinese Academy of Sciences

Jiandong He

Dalian Institute of Chemical Physics, Chinese Academy of Sciences

Sheng Ye

Dalian Institute of Chemical Physics, CAS

\section{Xiuli Wang}

Dalian Institute of Chemical Physics https://orcid.org/0000-0001-6231-4521

\section{Dongfeng Li}

Dalian Institute of Chemical Physics

\section{Heng Yin}

Dalian Institute of Chemical Physics, Chinese Academy of Sciences

Qianhong Zhu

Dalian Institute of Chemical Physics, Chinese Academy of Sciences

Huanwang Jing

College of Chemistry and Chemical Engineering, Lanzhou University

\section{Yuxiang Weng}

Chinese Academy of Sciences

\section{Fengtao Fan}

Dalian Institute of Chemical Physics

\section{Can Li ( $\nabla$ canli@dicp.ac.cn )}

Dalian Institute of Chemical Physics, Chinese Academy of Sciences https://orcid.org/0000-0002-93017850

\section{Article}

Keywords:

Posted Date: January 28th, 2022 
DOI: https://doi.org/10.21203/rs.3.rs-1275357/v1

License: (a) (i) This work is licensed under a Creative Commons Attribution 4.0 International License. Read Full License

Version of Record: A version of this preprint was published at Nature Communications on July 22nd, 2022. See the published version at https://doi.org/10.1038/s41467-022-32002-y. 


\section{Novel bipolar charge collecting structure enabling overall water splitting on ferroelectric photocatalysts}

Yong Liu ${ }^{l}$, Zhuan Wang ${ }^{3}$, Jiandong $\mathrm{He}^{l}$, Sheng $\mathrm{Ye}^{l}$, Xiuli Wang ${ }^{l}$, Dongfeng $\mathrm{Li}^{l}$, Heng Yin $^{l}$, Qianhong Zhu ${ }^{l}$, Huanwang Jing ${ }^{2}$, Yuxiang Weng ${ }^{3}$, Fengtao Fan ${ }^{l *}$, Can $\mathrm{Li}^{1,2 *}$

Dr. Y. Liu, J. He, Dr. S. Ye, Prof. X. Wang, D. Li, Dr. H. Yin, Q. Hong, Prof. F. Fan, Prof. C. Li

${ }^{1}$ State Key Laboratory of Catalysis, Dalian Institute of Chemical Physics, Chinese Academy of Sciences, Dalian National Laboratory for Clean Energy, Dalian 116023, China.

Email: ftfan@dicp.ac.cn, canli@dicp.ac.cn

Prof. H. Jing, Prof. C. Li

${ }^{2}$ State Key Laboratory of Applied Organic Chemistry, Advanced Catalysis Center, College of Chemistry and Chemical Engineering, Lanzhou University, Lanzhou 730000 , China

Dr. Z. Wang, Prof. Y. Weng

${ }^{3}$ The Laboratory of Soft Matter Physics, Beijing National Laboratory for Condensed Matter Physics, Institute of Physics Chinese Academy of Science, Beijing 100190, China 


\begin{abstract}
Due to the unidirectional charge separation and above-gap photovoltage, ferroelectrics have been considered as excellent photocatalytic candidates for solar fuel production. However, the performance of ferroelectric photocatalysts is often moderate. Few reports demonstrated that these kinds of photocatalyst could achieve overall water splitting. Here we propose a novel approach to fabricate interfacial charge collecting nano-structures on ferroelectric's positive and negative domains, enabling overall water splitting in ferroelectric photocatalysts. We observed efficient accumulations of photogenerated electrons and holes within their thermalization length (about $50 \mathrm{~nm}$ ) around the $\mathrm{Au}$ nanoparticles located in the positive and negative domains of $\mathrm{BaTiO}_{3}$ single crystal. Photocatalytic overall water splitting was firstly observed on ferroelectric $\mathrm{BaTiO}_{3}$ single crystal after assembly oxidation and reduction cocatalysts on the positive and negative charged Au nanoparticles. The idea of fabricating bipolar charge collecting structure on the ferroelectrics to achieve overall water splitting paves the new way for utilizing the energetic photogenerated charges in solar energy conversion.
\end{abstract}




\section{Introduction}

Ferroelectrics with switchable spontaneous polarization have shown tantalizing potential in memory storage and integrated microelectronics ${ }^{1-5}$. Meanwhile, power conversion efficiency exceeds unity, and large photovoltage above the bandgap have been reported in some ferroelectrics ${ }^{6-10}$. The photoelectric characters of ferroelectrics have drawn much interest in solar fuel production ${ }^{11-14}$. Compared to the traditional charge separation driving force via drift or diffusion mechanisms in common semiconductor photocatalyst, ferroelectric semiconductors possess charge separation driving force due to spontaneous polarization ${ }^{15-20}$. The unique characters in asymmetric crystals endows ferroelectric semiconductors the bulk photovoltaic effect (BPVE), favoring efficient photogenerated charge separation within the nonthermalizaion length $^{21,22}$. With these photogenerated charges, the Shockley-Queisser limit for the power conversion efficiency in the ferroelectric devices have been exceeded under one sun illumination (AM $1.5 \mathrm{G})^{6}$. Despite the enormous potential applications, the application of ferroelectrics in photovoltaic devices remains scarce. In particular, ferroelectric semiconductors are not yet reported for photocatalytic overall water splitting. Although they possess both spontaneous ferroelectric polarization induced internal field for massive charge separation and thermodynamically suitable energy band structure for overall water splitting.

The charge separation mechanisms in ferroelectric, known as BPVE, are often explained by two mechanisms: shift and ballistic ${ }^{23}$. The shift mechanism originates from a quantum phenomenon in the noncentrosymmetric crystal. It is the result of the coherent evolution of a quantum wave packet and the photoexcitation-induced shift of real space. The ballistic mechanism is related to the photogenerated, nonthermalized charges with asymmetric momentum distribution in the noncentrosymmetric crystal (FIG. 1a). The nonthermalized charges descend to the band bottom via a length $\mathrm{L}_{0}$, also called thermalization length. $\mathrm{L}_{0}$ depends on materials and incident photons in tens to hundreds of nanometers. Within $\mathrm{L}_{0}$, all the photogenerated charges contribute to BPVE and yield the highest solar energy conversion efficiency. Hexagonal close-packed 
metallic electrode arrays with accurate distance were predicted to have the highest collection and utilization of photogenerated charges (FIG. S1). Based on this principle, Spanier et al. prepare a device with a single-tip electrode contact and an array with 24 tips. The device generated a current density of $17 \mathrm{~mA} \mathrm{~cm}{ }^{-2}$ under the illumination of AM 1.5 $\mathrm{G}^{6}$. Photogenerated charges are concentrated around every individual tip and then collected via the ITO electrode. However, the fully-covered ITO electrode hinders the transmissivity in the ultraviolet range, where BTO has the most significant absorption coefficient. As a result, the performance of this device is mediocre and performs well below expectations. The utilization of photogenerated charges in ferroelectrics for high-efficiency solar energy conversion remains a longstanding challenge, despite the theoretical basis seeming quite clear. Thus, well-designed micro/nanostructures in ferroelectric-based semiconductors are of substantial importance in solar energy conversion. There left plenty of room for the explore of charge separation mechanisms at micro/nanoscale to achieve photocatalytic overall water splitting.

In this work, we proposed a novel approach to fabricate nano charge collecting structures at metal/ferroelectric interface to enable overall water splitting ability in ferroelectric photocatalysts, $\mathrm{Au}$ array patterned $\mathrm{BaTiO}_{3}$ single crystal. We find the anomalous concentration of photogenerated electrons and holes in Au particles, located at $+\mathrm{P}$ and $-\mathrm{P}$ domains in $\mathrm{BaTiO}_{3}$ single crystal, respectively. It is proved that the photogenerated charges are concentrated around $\mathrm{Au}$ particle within a hemisphere of radius $\mathrm{L}_{0}$, the thermalization length, about $50 \mathrm{~nm}$. Due to the energetic photogenerated charges, fabricated Au array/BTO photocatalysts show substantial photocatalytic overall water splitting performance. The measured thermalization length $\mathrm{L}_{0}$ is also the key experimental prescription in designing high efficiency ferroelectrics in solar energy conversion at nanoscales.

FIG. 1a shows our approach for high-efficiency solar energy conversion. In detail, Au particles in hexagonal arrays with proper density are fabricated on the surface of the ferroelectric semiconductor substrate. Individual Au particle is an enhanced charge 
collection and utilization point. And then, cocatalysts can be selectively deposited on $\mathrm{Au}$ particles under illumination. On the one hand, the concentrated photogenerated charges within thermalization length could promote photocatalytic activity. On the other hand, the photocatalytic reduction and oxidation reactions can be spatially separated on positive and negative polarization ferroelectric domains, respectively. In the case of photocatalytic water splitting, the hydrogen evolution reaction and oxygen evolution reaction can simultaneously occur. Solar energy conversion into chemical energy is feasible via overall water splitting.

Then, a typical model is established to clarify the behavior of photogenerated charges at the interface of metal and ferroelectrics. In detail, a (001)-oriented $\mathrm{BaTiO}_{3}$ single crystal is applied as ferroelectric substrate, where Au nanoparticles are dispersed. Au particles are about $200 \mathrm{~nm}$ in diameter and about $50 \mathrm{~nm}$ in thickness (FIG. 1b, FIG. $\mathrm{S} 1)$. Kelvin probe force microscopy (KPFM) is then applied to map the surface potential of Au/BTO under dark and light excitation conditions, as shown in FIG. 1c, d. Measured surface potential is the contact potential difference (CPD) between the AFM tip and the sample. And then, the CPD is converted to localized workfunction (LWF) for better understanding (Details in Experiments and Supporting Information). As shown in FIG. 1c and Line 1, the LWF of BTO at $-\mathrm{P}$ and $+\mathrm{P}$ ferroelectric domain is markedly different. At the $+\mathrm{P}$ BTO, BTO has downward surface band bending and the LWF is lower. On the contrary, BTO has upward surface band bending at the -P domain, and the LWF is higher. The polarization-induced surface contrast coincides with previous results ${ }^{24,25}$. It is noticeable that the LWF changes at the interface of $\mathrm{Au} / \mathrm{BTO}$ are more obvious (FIG. 1c and Line 3). At the Au/BTO interface in -P domain, the LWF of BTO is even higher, indicating that a Schottky-like junction with a depleting layer formed at the interface of Au/BTO. Because ferroelectric BTO is known as oxygen vacancies induced n-type semiconductor and Au possess large work function. Similarly, at the $+\mathrm{P} \mathrm{Au} / \mathrm{BTO}$ interface, the LWF of BTO is even lower due to the formation of a quasi-Ohmic contact and an accumulation layer. The LFW of Au/BTO in the dark 
confirms the formation of Schottky-like junction at $-\mathrm{P}$ and quasi-Ohmic contact at $+\mathrm{P}$ via KPFM, same as previous ferroelectric devices ${ }^{26-28}$.

The KPFM experiments are performed under $355 \mathrm{~nm}$ UV-light excitation (3.49 eV, about $0.5 \mathrm{~mW} / \mathrm{cm}^{2}$ ) to investigate the photogenerated charge separation. The photon energy is higher than the bandgap of BTO $\left(\mathrm{E}_{\mathrm{g}}=3.2 \mathrm{eV}\right)$, which is super-band illumination. The selected photon energy exceeds the bandgap of BTO but away from the surface plasmon resonance (SPR) excitation of Au particles at this size (SPR peak position at about $790 \mathrm{~nm})^{29}$. Thus, plasmon resonance absorption of Au would not affect BTO substrate at $355 \mathrm{~nm}$ UV illumination (FIG. S3). As shown in FIG. 1d, under the UV-light excitation, the LWF at the Au/BTO interface is significantly changed. And the WLF of the domain wall shifts about $0.34 \mathrm{eV}$ due to the bulk photovoltage effect (BPVE) of ferroelectric polydomain in the bulk of BTO (FIG. S7). And thus, the LWF at the domain wall between two antiparallel domains is taken as a reference ${ }^{25}$. The bar scale in both FIG. 1c and $1 \mathrm{~d}$ is $0.42 \mathrm{eV}$.

Then, a detailed analysis of the LWF line profiles taken across the LWF images (FIG. 1c and 1d) is described. Firstly, the energy-band diagram of bare BTO is analyzed. As shown in FIG. 1e, line 1 and line 2 are the LWF line profiles between two antiparallel domains in dark and under UV-light excitation, respectively. The contrast of LWF between the two antiparallel domains decreases from about $0.12 \mathrm{eV}$ to about $0.1 \mathrm{eV}$. The result confirms that both the ferroelectric polarization induced downward band bending at $+\mathrm{P}$ domain and upward band bending at $-\mathrm{P}$ domain are reduced due to the photogenerated charges transferring to the surface. It is worth noting that the measured domain contrast is much lower than the ideal value due to the screening charges ${ }^{24,30}$. Afterward, in analogy to bare BTO, the LWF line profiles across Au particles across different BTO domains are analyzed. The LWF values extracted across lines 3 and 4 (FIG. 1f) are displayed in FIG. 1f. Interestingly, under illumination, the LWF difference between the two Au particles is increased from about $0.18 \mathrm{eV}$ to about $0.28 \mathrm{eV}$. The result indicates that the built-in voltage of $\mathrm{Au} / \mathrm{BTO}$ interface at either $+\mathrm{P}$ or $-\mathrm{P}$ domain is further enhanced. In contrast, the built-in voltage at SCRs, such as bared BTO and 
common metal/semiconductor Schottky junction, always decreases under illumination $^{31,32}$. The enhanced built-in voltage at the two types of Au/BTO interface proves that the Schottky-like depleting layer at -P domain is further depleted and the quasi-Ohmic-like accumulation layer at $+\mathrm{P}$ domain is also further accumulated. The above results provide strong evidences that photogenerated charges are concentrated around $\mathrm{Au}$ particles in the SCRs, agreeing with Spanier's speculation ${ }^{6}$. In the surface SCRs of bare BTO and common semiconductors, the built-in voltage decrease under illumination (FIG. S4, S5, S6) ${ }^{31-34}$. This phenomenon at Au/BTO is quite anomalous, entirely different from common metal/semiconductor junction.

To obtain further information, a detailed quantitative analysis is carried out. As shown in FIG. 2, the LWF at Au/BTO interface is nonlinearly fitted with an exponential decay formula (Details in Supporting Information), giving built-in voltage $\varphi_{b i}$ and $\mathrm{SCR}$ width $\mathrm{L}$. At $+\mathrm{PAu} / \mathrm{BTO}$, the additional built-in voltage $\varphi_{b i}$ at $\mathrm{Au} / \mathrm{BTO}$ interface increases from $32.2 \mathrm{mV}$ in the dark to $78.5 \mathrm{mV}$ under UV light excitation. It should be mentioned that the $\varphi_{b i}$ is the additional built-in voltage of BTO, obtained by subtracting the surface potential of bare BTO from that of BTO at Au/BTO (FIG. S2). And thus, measured $\varphi_{b i}$ is smaller than the real built-in voltage at $\mathrm{Au} / \mathrm{BTO}$ heterojunction. And the additional space charge region (SCR) width decreases from $90.7 \mathrm{~nm}$ to $52.3 \mathrm{~nm}\left(\mathrm{~L}_{0}\right)$ in UV-light. The photogenerated electrons are concentrated around $\mathrm{Au}$ particles with a narrower hemisphere of radius $\mathrm{L}_{0}$ (FIG. 2c, left). The thermalization length or the mean free path for a hot photoexcited electron or hole $\mathrm{L}_{0}=$ $\mathrm{g}_{31} \mathrm{e}^{-1} \hbar \omega /\left(\Phi \xi^{\mathrm{ex}}\right)^{23}$. For bulk BTO single crystal, $\mathrm{g}_{31}=3 \times 10^{-9} \mathrm{~cm} \mathrm{~V} \mathrm{~V}^{-1}, \hbar \omega$ is the incident photon energy and $3.49 \mathrm{eV}(355 \mathrm{~nm})$ in our experiment, $\Phi$ is the quantum yield, $\xi^{\mathrm{ex}}$ is the photoexcitation asymmetry parameter and the max value of $\xi^{\text {ex }}$ is $10^{-2}-10^{-3}$. Because $\alpha_{\mathrm{BTO}} \approx 5-10 \mathrm{~cm}^{-1}$ at $\mathrm{h} \omega=3.06 \mathrm{eV}$, most of the light is absorbed in the crystal. So $\mathrm{L}_{0}$ is 10-100 $\mathrm{nm}$. Moreover, the excitation asymmetry parameter falls as the photon frequency increases. Previously experiment demonstrated that the $\mathrm{L}_{0}$ is about $100 \mathrm{~nm}$ when $\hbar \omega$ is $3.06 \mathrm{eV}^{6}$. Based on the above discussion, measured $50 \mathrm{~nm} \mathrm{~L}_{0}$ at $3.49 \mathrm{eV}$ is 
reasonable in this work. Measured $\mathrm{L}_{0}$ is an essential prescription in designing ferroelectric photovoltaic devices and photocatalysts.

More remarkable, the electric field intensity at $\mathrm{Au} / \mathrm{BTO}$ interface increases from about $3 \mathrm{kV} / \mathrm{cm}$ to about $15 \mathrm{kV} / \mathrm{cm}$, five times larger (FIG. S8). $15 \mathrm{kV} / \mathrm{cm}$ is almost one order of magnitude higher than that of common SCRs ${ }^{19}, 35-39$. The analogous phenomenon at $-\mathrm{P} \mathrm{Au} / \mathrm{BTO}$ is shown in FIG. S8b. It is estimated that the steady-state charges density at the interface increases about 2-3 orders of magnitude under illumination (FIG. S8c).

The concentration of photogenerated charges can be attributed to twofold factors, the enhanced electric field around Au particles and the oxygen vacancies in BTO (FIG. S10). Due to the constructed metal/ferroelectric junctions, an intense field is concentrated about $150 \mathrm{~nm}$ away from the margin of Au particles. Under light excitation, impact ionization of oxygen vacancies occurs within the enhanced electric field, as reported in the literature ${ }^{6,40}$. A photon produces the first pair of electron $\mathrm{e}_{1}$ and hole $\mathrm{h}_{1}$ from the oxygen vacancy. In this situation, $\mathrm{e}_{1}$ with high mobility relaxes and produces a second pair of electron $\mathrm{e}_{2}$ and hole $\mathrm{h}_{2}$. As a result, photogenerated charges are concentrated at the SCRs beneath BTO within a hemisphere of radius $\mathrm{L}_{0}$ about $50 \mathrm{~nm}$ around $\mathrm{Au}$.

To further confirm the charge transfer between ferroelectric BTO to Au, in situ photodeposition and KPFM experiments are performed. Two typical photodeposition reactions based on reduction (with photogenerated electrons) and oxidation (with photogenerated holes) reactions are carried out, under $355 \mathrm{~nm}$ UV-light excitation:

$$
\begin{gathered}
2 \mathrm{CrO}_{4}^{2-}+5 \mathrm{H}_{2} \mathrm{O}+6 e^{-} \rightarrow \mathrm{Cr}_{2} \mathrm{O}_{3} \downarrow+10 \mathrm{OH}^{-} \\
\mathrm{Mn}^{2+}+x \mathrm{H}_{2} \mathrm{O}+(2 x-2) \mathrm{h}^{+} \rightarrow \mathrm{MnO}_{x} \downarrow+2 x \mathrm{H}^{+}
\end{gathered}
$$

AFM topography in FIG. 3a shows that $\mathrm{CrO}_{4}{ }^{2-}$ is primarily reduced on $\mathrm{Au}$ particles to form a solid layer in $+\mathrm{P}$ domain, indicating the formation of $\mathrm{Cr}_{2} \mathrm{O}_{3}$ layer. The charge density of electrons on $\mathrm{Au}$ particles is higher than that of BTO. Thus, the $\mathrm{CrO}_{4}{ }^{2-}$ is primarily reduced to $\mathrm{Cr}_{2} \mathrm{O}_{3}$ on Au particles instead of ferroelectric BTO. Furthermore, the KPFM images before and after photodeposition are measured and shown in FIG. $3 b$ 
and 3c. The darker in the LWF images indicates higher LWF. After deposition, the photogenerated electrons remain concentrated around Au particles. The built-in voltage at $\mathrm{Au} / \mathrm{BTO}$ is further enhanced due to the deposition of $\mathrm{Cr}_{2} \mathrm{O}_{3}$. High-resolution Scanning Electron Microscope (HRSEM) image in FIG. 3d also shows a thin layer of $\mathrm{Cr}_{2} \mathrm{O}_{3}$ on the surface of $\mathrm{Au}$ particles. In contrast, as shown in FIG. $3 \mathrm{e}, \mathrm{Mn}^{2+}$ prefers to be oxidized to $\mathrm{MnO}_{\mathrm{x}}$ on Au particle under UV-light. At the same time, the KPFM images in FIG. $3 \mathrm{f}$ and $3 \mathrm{~g}$ indicate the charge separation at Au/BTO interface remains the same after photodeposition. HRSEM in FIG. 3h shows that $\mathrm{MnO}_{\mathrm{x}}$ prefers to deposit on Au particle. The above in situ photodeposition experiments validate the proposed model that the photogenerated electrons and holes are separately collected by the $\mathrm{Au}$ particles in $+\mathrm{P}$ and $-\mathrm{P}$ domains of BTO.

Ferroelectric photocatalyst is then designed based on the above experiment phenomenon and measured essential experimental prescription. Except for the thermalization length of BTO, several other factors should also be concerned, such as array density, Au particle size. The work function, metal-ferroelectric interface, and surface plasmon resonance (SPR) of Au particles are pronouncedly size-dependent ${ }^{41,42}$. Despite the Au array's density decrease with Au particle size, large Au particles with higher charge capacity, better metal/ ferroelectric interface, and red-shift SPR are preferred. Besides, the electric field around the charged Au particle arrays should also be well considered. Thus, the distance between the margin of two adjacent Au particles should be more than twice $\mathrm{L}_{0}$ due to the electrostatic repulsion between them (FIG. S18 and S19). Based on these aspects, appropriately designed ferroelectric photocatalysts are shown in FIG. 4a. Periodic hexagonal close-packed (hcp) Au particles on BTO are prepared with self-assemble polystyrene microsphere template (Details in Supporting Information, FIG. S12-16). The Au particles are about 200-230 nm in diameter with $500 \mathrm{~nm}$ periodicity. The distance between the margin of two adjacent Au particles is about 250-300 $\mathrm{nm}$. The electric field simulation indicates that the electric field surrounding Au array is massively enhanced and radially expands, but different from an individual one. The enhanced field around the center Au particle is almost a 
hemisphere and contracted horizontally compared with individual one due to the electrostatic repulsion between the neighbor Au particles. The enhanced field extends about $80 \mathrm{~nm}$ from the margin of Au particle. A nonenhanced area is also found between the two $\mathrm{Au}$ particles due to the electrostatic repulsion. When the distance between the margin of two adjacent Au particles is $100 \mathrm{~nm}$, i.e., twice of $\mathrm{L}_{0}$, the periodicity decreases to $300 \mathrm{~nm}$. The strong electrostatic repulsion between the neighbor Au particles enables a shrunken and reduced electric field (FIG. S19). The electric field extends less than the $\mathrm{L}_{0}$ and cannot conform to the demand of charge collection within $\mathrm{L}_{0}$. Based on the simulation results, we demonstrate that the designed Au array on BTO is rational.

Furthermore, X-ray photoelectron spectroscopy (XPS) is conducted to investigate the interfacial contact between Au array and BTO. As shown in FIG. 4b, several peaks are layered together and can be figured as $4 \mathrm{~d}$ peaks of $\mathrm{Ba}^{2+}$ and $4 \mathrm{f}$ peaks of $\mathrm{Au}^{0}$. These peaks can fit certain constraints, such as area ratio and characterized peak location (Details in Supporting Information). Both the $\mathrm{Au}^{0} 4 \mathrm{f}_{7 / 2}$ and $\mathrm{Au}^{0} 4 \mathrm{f}_{5 / 2}$ peaks are divided into two peaks. The binding energy (BE) between the two peaks is about $0.6 \mathrm{eV}$. Because the $\mathrm{BE}$ difference between the $\mathrm{Au}^{0}$ and $\mathrm{Au}^{1+}$ is usually about $1.5 \mathrm{eV}$. And it is the same with $\mathrm{Au}^{1+}$ and $\mathrm{Au}^{3+}$. Thus, the $\mathrm{Au}$ element is in the chemical state of $\mathrm{Au}^{0}$ but different charge density due to the constructed heterojunction with BTO. In detail, the higher $\mathrm{BE}$ of $\mathrm{Au}^{0} 4 \mathrm{f}$ can be assigned to the $\mathrm{Au}$ particle at $+\mathrm{P}$ with quasi-Ohmic contact. Analogously, the lower $\mathrm{BE}$ of $\mathrm{Au}^{0} 4 \mathrm{f}$ can be assigned to the $\mathrm{Au}$ particle at $-\mathrm{P}$ with Schottky contact. Notably, the electrostatic charges on Au particles are the requirement for the enhanced electric field at Au/BTO interface. Both the XPS and KPFM results confirm the well-fabricated metal/ferroelectric heterojunctions and the polydomain ferroelectric structure of BTO crystal. And thus, the reduction and oxidation reactions can be simultaneously achieved and spatially separated on the surface of the BTO crystal.

Hydrogen evolution reaction in FIG. 4c demonstrates that the Au/BTO exhibits significantly higher activity than bare BTO, providing further experimental verification of the collecting and utilizing photogenerated charges at metal/ferroelectric interface. 
After selectively photodeposition $\left(\mathrm{Rh} / \mathrm{Cr}_{2} \mathrm{O}_{3} \text { and } \mathrm{CoOOH}\right)^{43,44}$, the overall water splitting is achieved in pure water (FIG. 4d). This could be the first case in the literature that ferroelectric structures can split pure water via photocatalysis. Even though perovskite BTO possesses both thermodynamically suitable energy band and massive charge separation driving force for water splitting, the overall water splitting of BTO is still not reported yet. After constructing nanostructures to collect and utilize the photogenerated charges, we successfully demonstrate that the overall water splitting in pure water can be achieved. These results emphasize the significance of utilizing the photogenerated charges in ferroelectrics within the thermalization length.

\section{Conclusions}

In summary, we have shown that the overall photocatalytic water splitting can be achieved in ferroelectric photocatalysts via collecting and utilizing the photogenerated charges within the thermalization length in a prototype of Au/BTO photocatalysis. Using KPFM, we have observed the concentration of photogenerated charges within the thermalization length of BTO at the Au/BTO interface. Measured thermalization length is an essential experimental prescription for fabricating high-efficiency photocatalytic and photovoltaic devices at the nanoscale. With this novel structure design, constructed ferroelectric photocatalysts can perform photocatalytic overall water splitting. The experimental design definitely opens a paradigm of designing the ferroelectric photocatalysts for efficient solar energy conversion. 

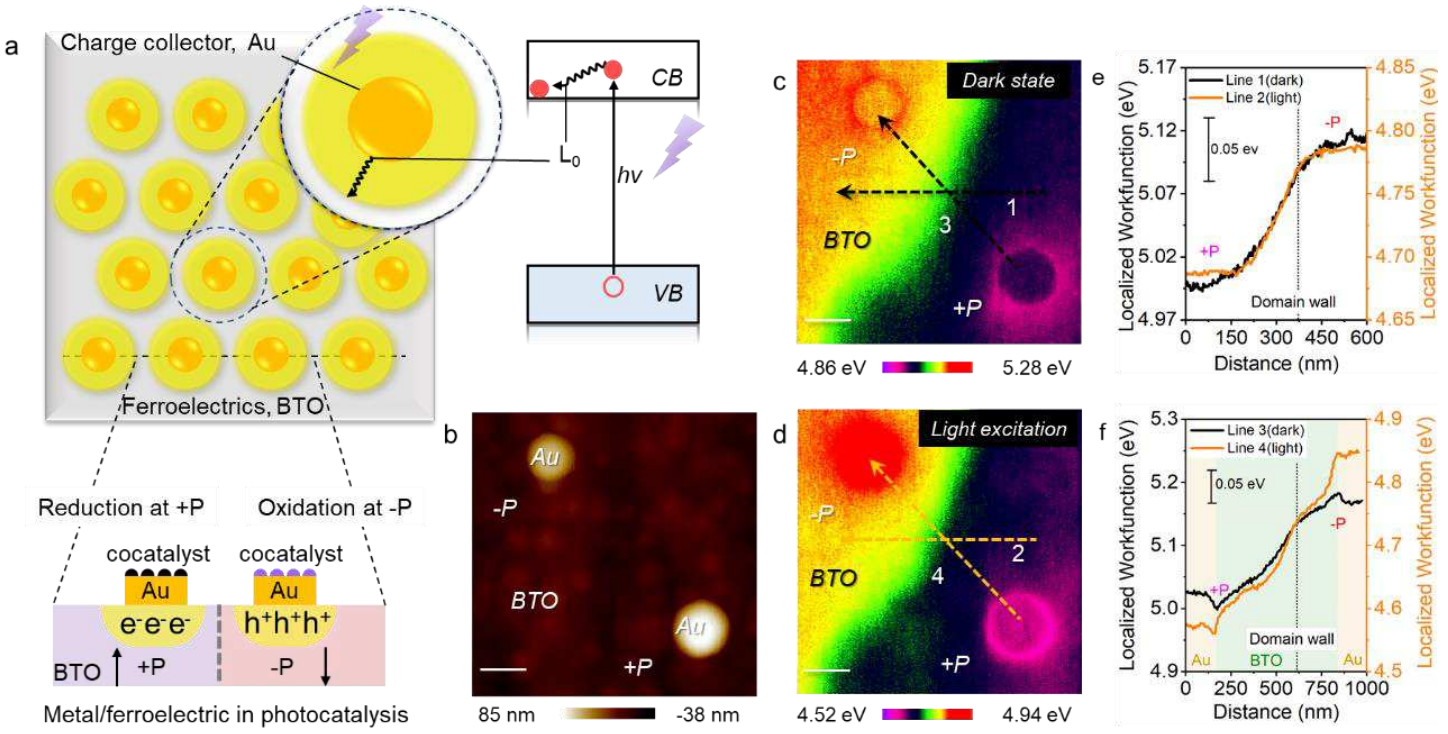

FIG. 1 (a) Schematics of proposed metal/ferroelectric photocatalyst. (b) AFM topography of $\mathrm{Au}$ particles on $\mathrm{BaTiO}_{3}$ single crystal. Scale bar, $200 \mathrm{~nm}$. (c) LWF of Au/BTO in the dark. Scale bar, $200 \mathrm{~nm}$. (d) LWF of Au/BTO under $355 \mathrm{~nm}$ UV-light $\left(0.5 \mathrm{~mW} / \mathrm{cm}^{2}\right)$. Scale bar, $200 \mathrm{~nm}$. (e) Line 1 (dark) and 2 (UV-light) profile images were taken across two antiparallel ferroelectric domains of BTO. (f) Line 3 (dark) and 4 (UV-light) profile images were taken across two antiparallel ferroelectric domains of $\mathrm{Au} / \mathrm{BTO}$. 
a

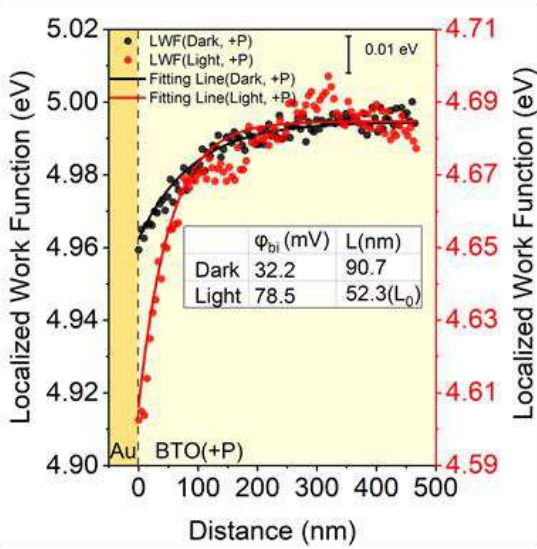

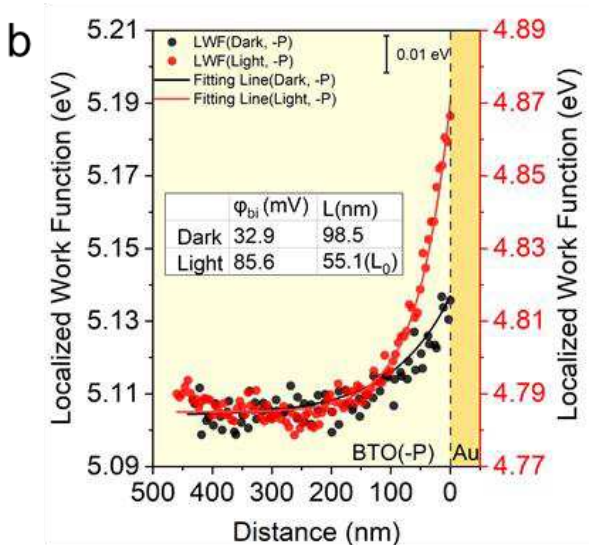

Distance $(\mathrm{nm})$

C

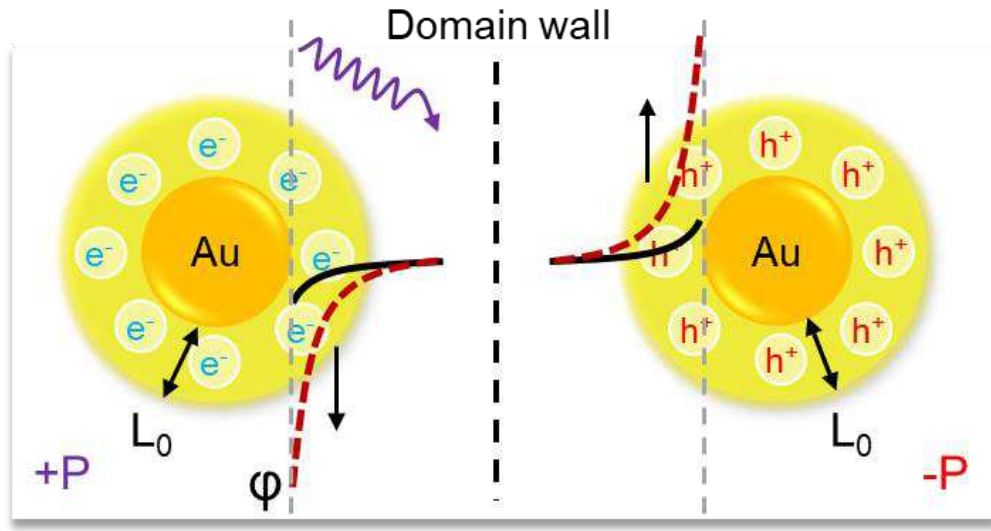

FIG. 2 (a) LWF and fitting line at the interface of $+\mathrm{P}$ Au/BTO in the dark (black) and $355 \mathrm{~nm}$ UV-light (red). (b) LWF and fitting line at the interface of -P Au/BTO in the dark (black) and $355 \mathrm{~nm}$ UV-light (red). (c) Diagram of charge separation of Au/BTO at $+\mathrm{P}($ left $)$ and $-\mathrm{P}($ right $)$ in dark(black solid line) and light(red dashed line). 
a

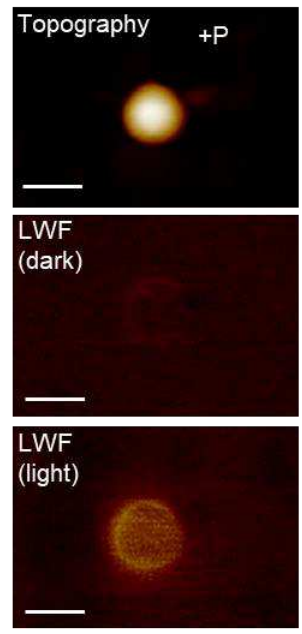

d

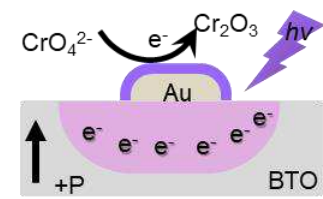

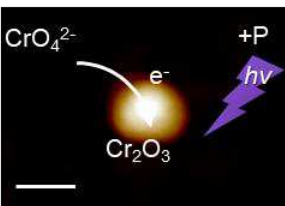
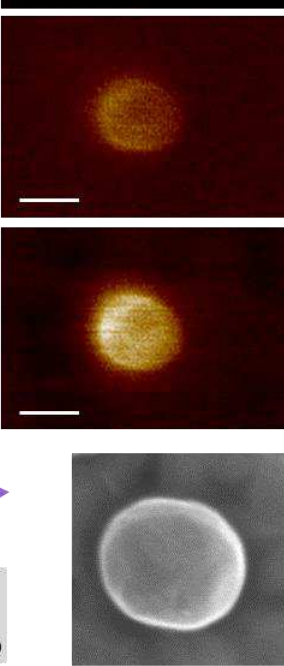
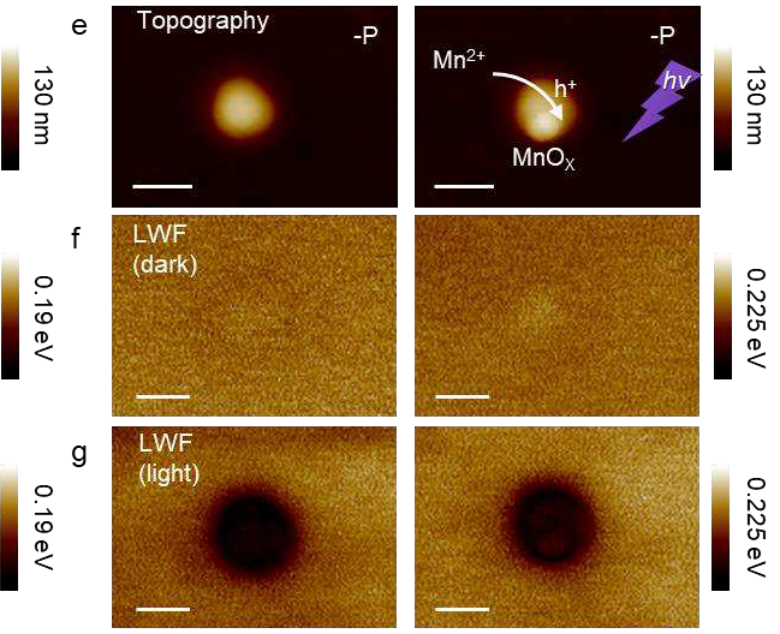

$\mathrm{h}$

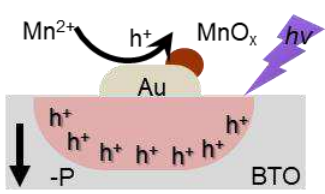

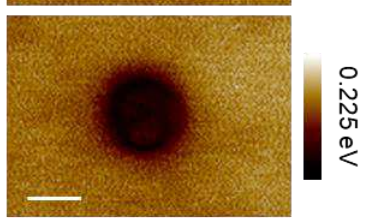

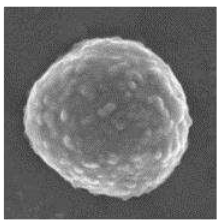

FIG. 3 (a-d) Photoreduction on Au at + P BTO. (a) AFM topography of Au/BTO at + P before (left) and after (right) photodepostion. (b) LWF in dark before (left) and after (right) photodepostion. The darker indicates the higher LWF. (c) LWF in light before (left) and after (right) photodepostion. (d) Schematic illustrations of photoreduction of $\mathrm{Cr}_{2} \mathrm{O}_{3}$ on $\mathrm{Au}$ at $+\mathrm{P}$. (e-h) Photooxidation on Au at -P BTO. (e) AFM topography of $\mathrm{Au} / \mathrm{BTO}$ at -P before (left) and after (right) photodepostion. (f) LWF in dark before (left) and after (right) photodepostion. The darker indicates the higher LWF. (g) LWF in light before (left) and after (right) photodepostion. (h) Schematic illustrations of photooxidation of $\mathrm{MnO}_{\mathrm{x}}$ on $\mathrm{Au}$ at -P. Scale bar, $200 \mathrm{~nm}$. 
a
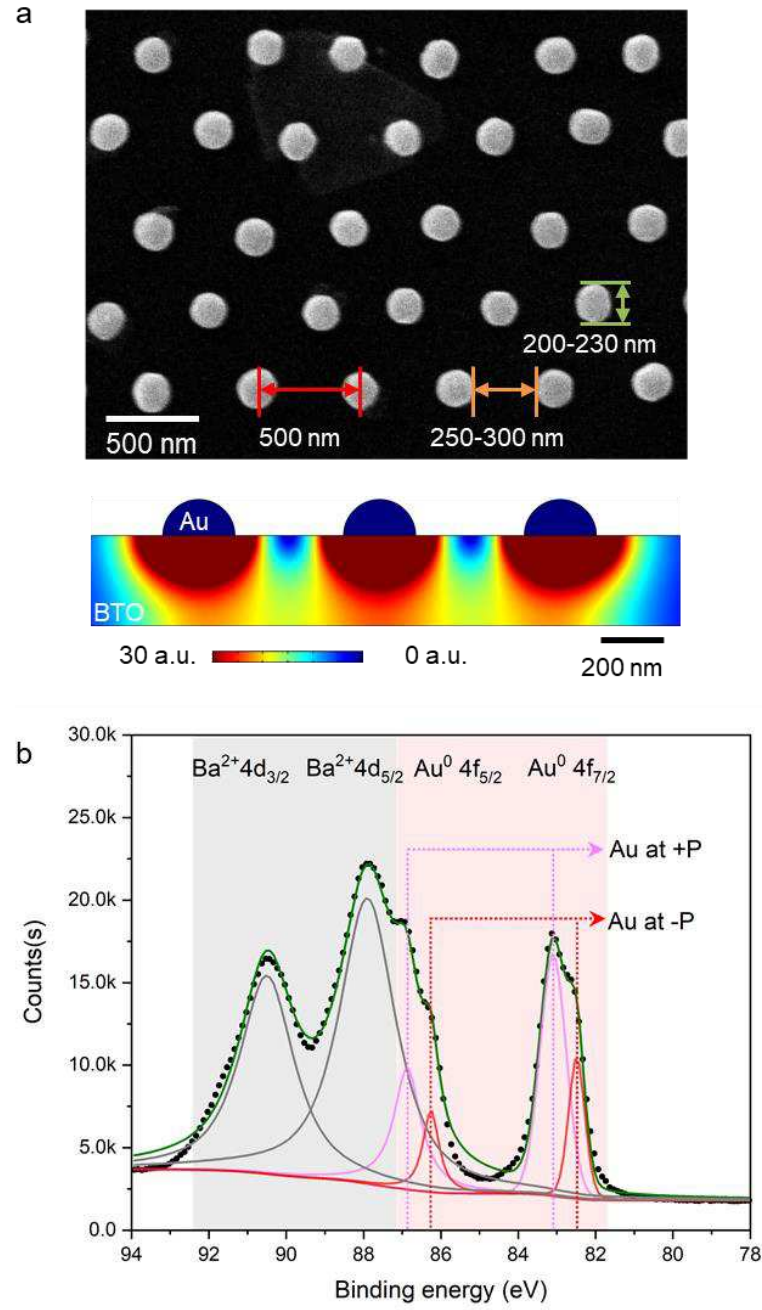

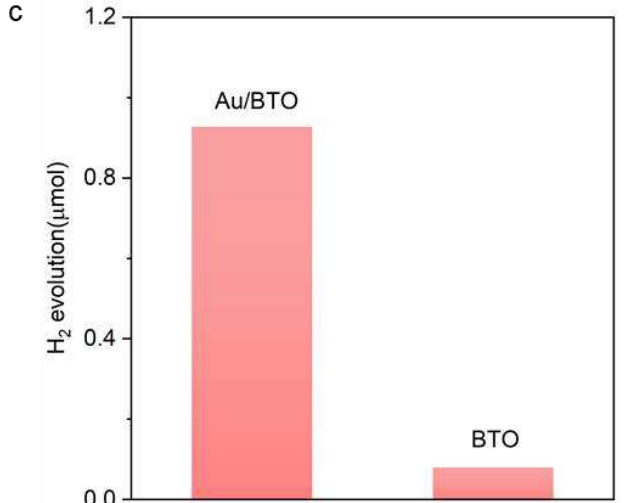

d
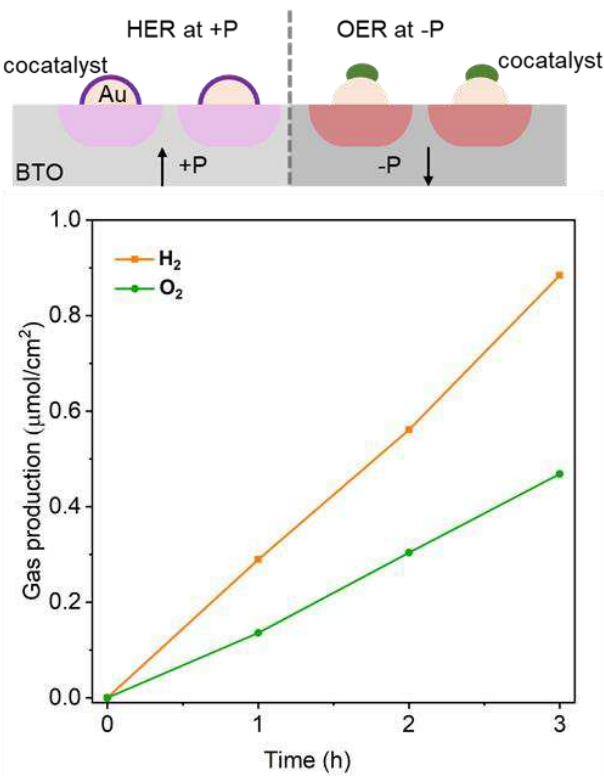

FIG. 4 (a) SEM image of Au array on BTO single crystal (upper) and simulated electric field intensity distribution of Au array/BTO (lower). (b) High-resolution XPS profiles of Au array/BTO. (c) Hydrogen evolution reaction of Au array/BTO and BTO. (d) Overall water splitting reactions of Au array/BTO with cocatalysts in pure water. 


\section{Supporting Information}

Supporting Information is available from the $\mathrm{xxx}$ or from the author.

\section{Acknowledgments}

This work was conducted by the Fundamental Research Center of Artificial Photosynthesis (FReCAP) and financially supported by the National Natural Science Foundation of China $(22088102,22102173)$, CAS Projects for Young Scientists in

Basic Research (YSBR-004), National Key R\&D Program of China (2021YFA1500600) and Dalian Institute of Chemical Physics Innovation Foundation (DICPSZ201801). Fellowship of China Postdoctoral Science Foundation, Grant No. 2020M690041.

\section{Author contributions}

Y.L. performed the experiments and wrote the paper. Z. W. and Y. W. performed the fsTAS experiments. X. W, D. L, and H. Y. performed the analysis of fs-TAS measurements.S.Y. and J.H. performed the photocatalytic experiments. Q. Z. and H.J. performed the analysis of KPFM measurements. F.F. and C.L. analyzed data and revised the manuscript.

\section{Competing interests}

The authors declare no competing financial interests and no competing non-financial interests.

Received: ((will be filled in by the editorial staff))

Revised: ((will be filled in by the editorial staff))

Published online: ((will be filled in by the editorial staff))

\section{Reference:}

1. Wang S, et al. Two-dimensional ferroelectric channel transistors integrating ultrafast memory and neural computing. Nat Commun 12, 53 (2021).

2. Chai X, et al. Nonvolatile ferroelectric field-effect transistors. Nat Commun 11, 2811 (2020).

3. Dawber M, Rabe K, Scott J. Physics of thin-film ferroelectric oxides. Rev Mod Phys 77, 1083 (2005).

4. A century of ferroelectricity. Nat Mater 19, 129 (2020). 
5. Wang X, et al. Van der Waals engineering of ferroelectric heterostructures for longretention memory. Nat Commun 12, 1109 (2021).

6. Spanier JE, et al. Power conversion efficiency exceeding the Shockley-Queisser limit in a ferroelectric insulator. Nat Photon 10, 611 (2016).

7. Yang SY, et al. Above-bandgap voltages from ferroelectric photovoltaic devices. Nat Nanotech 5, 143 (2010).

8. Xiao Z, et al. Giant switchable photovoltaic effect in organometal trihalide perovskite devices. Nat Mater 14, 193 (2015).

9. Alexe M, Hesse D. Tip-enhanced photovoltaic effects in bismuth ferrite. Nat Commun 2, 256 (2011).

10. Nechache R, et al. Bandgap tuning of multiferroic oxide solar cells. Nat Photon 9, 61 (2014).

11. Liu G, et al. Selective Chemical Epitaxial Growth of $\mathrm{TiO}_{2}$ Islands on Ferroelectric $\mathrm{PbTiO}_{3}$ Crystals to Boost Photocatalytic Activity. Joule 2, 1095 (2018).

12. Zhen C, Yu JC, Liu G, Cheng HM. Selective deposition of redox co-catalyst(s) to improve the photocatalytic activity of single-domain ferroelectric $\mathrm{PbTiO}_{3}$ nanoplates. Chem Commun 50, 10416 (2014).

13. Li S, et al. Epitaxial $\mathrm{Bi}_{2} \mathrm{FeCrO}_{6}$ Multiferroic Thin Film as a New Visible Light Absorbing Photocathode Material. Small 11, 4018 (2015).

14. Su R, et al. Silver-modified nanosized ferroelectrics as a novel photocatalyst. Small 11, 202 (2015).

15. Khaselev O, Turner JA. A Monolithic Photovoltaic-Photoelectrochemical Device for Hydrogen Production via Water Splitting. Science 280, 425 (1998).

16. Jiang T, Xie T, Yang W, Chen L, Fan H, Wang D. Photoelectrochemical and Photovoltaic Properties of $\mathrm{p}-\mathrm{n} \mathrm{Cu}_{2} \mathrm{O}$ Homojunction Films and Their Photocatalytic Performance. J Phys Chem C 117, 4619 (2013).

17. Wang X, et al. Photocatalytic overall water splitting promoted by an alpha-beta phase junction on $\mathrm{Ga}_{2} \mathrm{O}_{3}$. Angew Chem Int Ed 51, 13089 (2012).

18. Tian G, Fu H, Jing L, Xin B, Pan K. Preparation and characterization of stable biphase $\mathrm{TiO}_{2}$ photocatalyst with high crystallinity, large surface area, and enhanced photoactivity. J Phys Chem C 112, 3083 (2008).

19. Chen RT, et al. Charge separation via asymmetric illumination in photocatalytic $\mathrm{Cu}_{2} \mathrm{O}$ particles. Nat Energy 3, 655 (2018).

20. Li L, et al. Sub-10 nm rutile titanium dioxide nanoparticles for efficient visiblelight-driven photocatalytic hydrogen production. Nat Commun 6, 5881 (2015).

21. Fridkin VM. Bulk photovoltaic effect in noncentrosymmetric crystals. Crystallogr Rep 46, 654 (2001).

22. Fridkin VM. Boltzmann principle violation and bulk photovoltaic effect in a crystal without symmetry center. Ferroelectrics 503, 15 (2016).

23. Sturman BI, Fridkin VM. Photovoltaic and Photo-refractive Effects in Noncentrosymmetric Materials. CRC Press (1992).

24. Kalinin SV, Bonnell DA. Local potential and polarization screening on ferroelectric surfaces. Phys Rev B 63, 125411 (2001).

25. Kalinin SV, Johnson CY, Bonnell DA. Domain polarity and temperature induced 
potential inversion on the BaTiO3(100) surface. J Appl Phys 91, 3816 (2002).

26. Wang C, et al. Switchable diode effect and ferroelectric resistive switching in epitaxial $\mathrm{BiFeO}_{3}$ thin films. Appl Phys Lett 98, 192901 (2011).

27. Yi HT, Choi T, Choi SG, Oh YS, Cheong SW. Mechanism of the switchable photovoltaic effect in ferroelectric $\mathrm{BiFeO}_{3}$. Adv Mater 23, 3403 (2011).

28. Lee D, et al. Polarity control of carrier injection at ferroelectric/metal interfaces for electrically switchable diode and photovoltaic effects. Phys Rev B 84, 125305 (2011).

29. Yin $\mathrm{H}$, et al. Plasmonic and sensing properties of vertically oriented hexagonal gold nanoplates. Nanoscale 10, 15058 (2018).

30. Gao P, et al. Atomic mechanism of polarization-controlled surface reconstruction in ferroelectric thin films. Nat Commun 7, 11318 (2016).

31. Singh Pratiyush A, et al. High responsivity in molecular beam epitaxy grown $\beta$ Ga2O3 metal semiconductor metal solar blind deep-UV photodetector. Appl Phys Lett 110, 221107 (2017).

32. Lu MY, Lu MP, You SJ, Chen CW, Wang YJ. Quantifying the barrier lowering of ZnO Schottky nanodevices under UV light. Sci Rep 5, 15123 (2015).

33. Zhou J, et al. Gigantic enhancement in response and reset time of $\mathrm{ZnO} \mathrm{UV}$ nanosensor by utilizing Schottky contact and surface functionalization. Appl Phys Lett 94, 191103 (2009).

34. Lu M-Y, Lu M-P, Chung Y-A, Chen M-J, Wang ZL, Chen L-J. Intercrossed SheetLike Ga-Doped ZnS Nanostructures with Superb Photocatalytic Actvitiy and Photoresponse. J Phys Chem C 113, 12878 (2009).

35. Zhu J, Fan FT, Chen RT, An HY, Feng ZC, Li C. Direct Imaging of Highly Anisotropic Photogenerated Charge Separations on Different Facets of a Single $\mathrm{BiVO}_{4}$ Photocatalyst. Angew Chem Int Ed 54, 9111 (2015).

36.Gao Y, et al. Directly Probing Charge Separation at Interface of $\mathrm{TiO}_{2} \mathrm{Phase}_{\text {Junction. }}$ J Phys Chem Lett 8, 1419 (2017).

37. Zhu J, et al. Visualizing the Nano Cocatalyst Aligned Electric Fields on Single Photocatalyst Particles. Nano Lett 17, 6735 (2017).

38. Chen R, Pang S, An H, Dittrich T, Fan F, Li C. Giant defect-induced effects on nanoscale charge separation in semiconductor photocatalysts. Nano Lett 19, 426 (2018).

39. Liu Y, et al. Internal-Field-Enhanced Charge Separation in a Single-Domain Ferroelectric $\mathrm{PbTiO}_{3}$ Photocatalyst. Adv Mater 32, 1906513 (2020).

40. Werner JH, Brendel R, Queisser HJ. Radiative efficiency limit of terrestrial solar cells with internal carrier multiplication. Appl Phys Lett 67, 1028 (1995).

41. Zhang Y, et al. Sensing the charge state of single gold nanoparticles via work function measurements. Nano Lett 15, 51 (2015).

42. Hou J, Nonnenmann SS, Qin W, Bonnell DA. A transition in mechanisms of size dependent electrical transport at nanoscale metal-oxide interfaces. Appl Phys Lett 103, 252106 (2013).

43. Takata $\mathrm{T}$, et al. Photocatalytic water splitting with a quantum efficiency of almost unity. Nature 581, 411 (2020). 
44. Kibria MG, et al. Tuning the surface Fermi level on p-type gallium nitride nanowires for efficient overall water splitting. Nat Commun 5, 3825 (2014). 


\section{Supplementary Files}

This is a list of supplementary files associated with this preprint. Click to download.

- AuarrayBTOSItxt.pdf 